Article

\title{
Quantifying Waterfowl Numbers: Comparison of Drone and Ground-Based Survey Methods for Surveying Waterfowl on Artificial Waterbodies
}

\author{
Shannon J. Dundas ${ }^{1, *}$, Molly Vardanega ${ }^{1}$, Patrick $\mathrm{O}^{\prime}$ Brien $^{2}$ and Steven R. McLeod ${ }^{1}$ \\ 1 Vertebrate Pest Research Unit, NSW Department of Primary Industries, 1447 Forest Rd, \\ Orange, NSW 2800, Australia; molly.vardanega@dpi.nsw.gov.au (M.V.); \\ steven.mcleod@dpi.nsw.gov.au (S.R.M.) \\ 2 Game Licensing Unit, NSW Department of Primary Industries, 620 Macauley St, \\ Albury, NSW 2640, Australia; patrick.o'brien@dpi.nsw.gov.au \\ * Correspondence: shannon.dundas@dpi.nsw.gov.au
}

check for updates

Citation: Dundas, S.J.; Vardanega, M.; O’Brien, P.; McLeod, S.R. Quantifying Waterfowl Numbers: Comparison of Drone and

Ground-Based Survey Methods for Surveying Waterfowl on Artificial Waterbodies. Drones 2021, 5, 5. https://doi.org/10.3390/drones 5010005

Received: 14 November 2020 Accepted: 20 December 2020 Published: 13 January 2021

Publisher's Note: MDPI stays neutral with regard to jurisdictional clai$\mathrm{ms}$ in published maps and institutional affiliations.

Copyright: $(\odot 2021$ by the authors. Licensee MDPI, Basel, Switzerland. This article is an open access article distributed under the terms and conditions of the Creative Commons Attribution (CC BY) license (https:// creativecommons.org/licenses/by/ $4.0 /)$.

\begin{abstract}
Drones are becoming a common method for surveying wildlife as they offer an aerial perspective of the landscape. For waterbirds in particular, drones can overcome challenges associated with surveying locations not accessible on foot. With the rapid uptake of drone technology for bird surveys, there is a need to compare and calibrate new technologies with existing survey methods. We compared waterfowl counts derived from ground- and drone-based survey methods. We sought to determine if group size and waterbody size influenced the difference between counts of nonnesting waterfowl and if detection of species varied between survey methods. Surveys of waterfowl were carried out at constructed irrigation dams and wastewater treatment ponds throughout the Riverina region of New South Wales (NSW), Australia. Data were analyzed using Bayesian multilevel models (BMLM) with weakly informative priors. Overall, drone-derived counts of waterfowl were greater $(+36 \%)$ than ground counts using a spotting scope $\left(\beta\right.$-ground $=0.64[0.62-0.66],\left(R^{2}=0.973\right)$ ) Ground counts also tended to underestimate the size of groups. Waterbody size had an effect on comparative counts, with ground counts being proportionally less than drone counts (mean $=0.74)$. The number of species identified in each waterbody type was similar regardless of survey method. Drone-derived counts are more accurate compared to traditional ground counts, but drones do have some drawbacks including initial equipment costs and time-consuming image or photo processing. Future surveys should consider using drones for more accurately surveying waterbirds, especially when large groups of birds are present on larger waterbodies.
\end{abstract}

Keywords: population estimates; UAV; Australian ducks

\section{Introduction}

The use of drones or unmanned aerial vehicles (UAVs) for wildlife surveys has increased greatly with an aerial perspective allowing for better visualization of wildlife across the landscape. Regular and accurate monitoring of animals to establish population estimates is essential for conservation work of any species [1,2] and the development of drone technology has improved the scope for quantifying wildlife populations. Estimates of population size can be particularly challenging for bird species, especially when birds flock in large numbers. Drones have been successfully used to assess glossy ibis (Plegadis falcinellus) breeding populations [3] and for quantifying nest numbers for breeding wood storks (Mycteria americana) [4], common terns (Sterna hirundo) [5], and lesser black-backed gulls (Larus fuscus) [6]. Drone technology is constantly evolving in terms of capability with extended battery life and improvements to image quality. The use of drones to survey wildlife therefore also needs to be adaptive as it evolves and survey techniques improve [1,7]. It is also important to compare and calibrate these new techniques with traditional survey methods. 
Waterbirds residing in and around water offer an ideal situation for using drones as a survey method. Large flocks of birds can be challenging to survey, especially in the case where they are sensitive to any human interaction or where animals may be located in inaccessible areas [8]. Often ground surveys can be hindered by limited visibility and restricted site access so an aerial view of the landscape has major advantages when surveying birds [9]. The use of drones also has the potential to eliminate human-related factors such as fatigue and distractions which can occur when conducting ground counts in the field. The collection of video and still images in the field allows for follow-up counts to be carried out back in the office. Videos and images can be paused, zoomed, and replayed to ensure all birds are identified and counted. Post-processing is usually the most timeconsuming part of drone surveys, but the development of automated image recognition to reduce processing times of images and videos is an active area of research [10,11].

We compared counts of nine native Australian waterfowl using two survey methods; ground-based and drone-based. Our research aims were to determine if: 1 . Drone counts were significantly different to ground counts, 2 . group size influences the difference between drone and ground counts, 3 . waterbody type influences the difference between drone and ground counts, and 4 . the number of species detected at a waterbody varies between survey methods.

\section{Materials and Methods}

\subsection{Study Area}

Surveys were carried out on constructed irrigation dams and wastewater treatment ponds within the Riverina region of New South Wales, Australia. Productive agriculture covers $\sim 91,000 \mathrm{~km}^{2}$ and is a well-established region for commercial farming of rice, cotton, grain, hay, oilseeds, legumes, citrus, wine grapes, tree nuts, and livestock for meat, milk, and wool [12]. We focused on waterfowl using irrigation dams and wastewater treatment ponds varying in size from 2-156+ ha. Surveys of waterfowl are carried out annually in this region to establish population estimates for the allocation of a pest mitigation harvest of waterfowl impacting on rice crops [13]. Selection of surveyed waterbodies was based on water presence, the presence of waterfowl, access permission from landowners, and within airspace not intercepted by airport landing and takeoff zones.

\subsection{Ground Surveys}

Nine species of waterfowl were the focus of these surveys: Grey Teal (Anas gracilis), Australian Wood Duck (Chenonetta jubata), Pacific Black Duck (Anas superciliosa), Hardhead (Aythya australis), Chestnut Teal (Anas castanea), Pink-eared Duck (Malacorhynchus membranaceus), Australasian Shelduck (Tadorna tadornoides), Blue-winged Shoveler (Anas rhynchotis), and Plumed Whistling Duck (Dendrocygna eytoni). Ground surveys were conducted by a single observer (S.J.D., M.V., or P.O.B.) at each waterbody using a spotting scope (Kowa Promina TSN-663, Kowa Company Limited, Japan or Swarovski ATX 25-60 $\times 85 \mathrm{~mm}$, Swarovski Optik, Austria) mounted to a tripod (Manfrotto 128RC, Manfrotto Distribution, Cassola, Italy). Ground counts were carried out immediately prior to flying the drone. All observers had experience identifying and counting waterfowl. The ground observer slowly scanned the entire dam from left to right and recorded the number and species of ducks seen into a voice recorder representing a total count for the dam.

\subsection{Drone Surveys}

For the drone surveys in March and August 2018, we used a multirotor Phantom 4 Pro drone (DJI, Shenzhen, China) to carry out drone surveys. The Pro version of the Phantom 4 has a 20-MP camera and can record high resolution video footage in $4 \mathrm{~K}$ at 60 frames per second (fps) with a battery life of $\sim 15 \mathrm{~min}$. We found the faster fps greatly improved our ability to identify waterfowl species from video (compared to $30 \mathrm{fps}$ for the standard DJI Phantom 4). This was especially noticeable when flying higher in an effort to reduce disturbance to waterfowl. For the 2019 and 2020 surveys, we upgraded to a larger 
multirotor drone, the Matrice 210 V2 with a Zenmuse X5S camera (DJI, Shenzhen, China) which records $4 \mathrm{~K}$ video at the required $60 \mathrm{fps}$ with a battery life of $\sim 25 \mathrm{~min}$.

Given the dynamic nature of waterfowl (flying, diving, swimming along the water, resting on the bank), videos were found to be better than still images for identifying duck species. To ensure a consistent flight path and to ensure all waterfowl present on the dams were captured on the video, we used an automated flight program, Autopilot (Hangar Technology, Austin, TX, USA) for the Phantom 4 drone. For the Matrice 210, we used the recommended DJI software, DJI Pilot, to run automated flights. In both programs, we flew a grid pattern with the flight direction altered for each dam in order to minimize glare off the water. We aimed to collect video footage of waterfowl across each dam while they were on the water by flying the drone as low as possible without causing them to fly away from the waterbody. This was necessary to collect adequate imagery to identify waterfowl to species. Depending on the reaction of waterfowl to the drone, the survey height of the drone varied between 18-35 m. We also found flying the drone at a faster speed (20-25 kph) reduced the amount of disturbance to waterfowl sitting along banks or on water as the drone moves past the birds before they notice its presence and react. We found that the short-term disturbance caused by a quick drone flyover caused no more distance to waterfowl than being approached on ground by an observer. All birds were in a non-breeding state, so short-term disturbance causing ducks to fly or dive was unlikely to cause any adverse long-term effects. The angle of the camera was set to face downwards $45^{\circ}$ which gave a better view of the ducks from the side and above (Figure 1 ). The drone was generally launched $>500 \mathrm{~m}$ away from birds. We avoided flying during adverse weather such as stormy overcast conditions, windy conditions more than a medium breeze, and drizzly rain as these conditions greatly reduced the quality of the video.

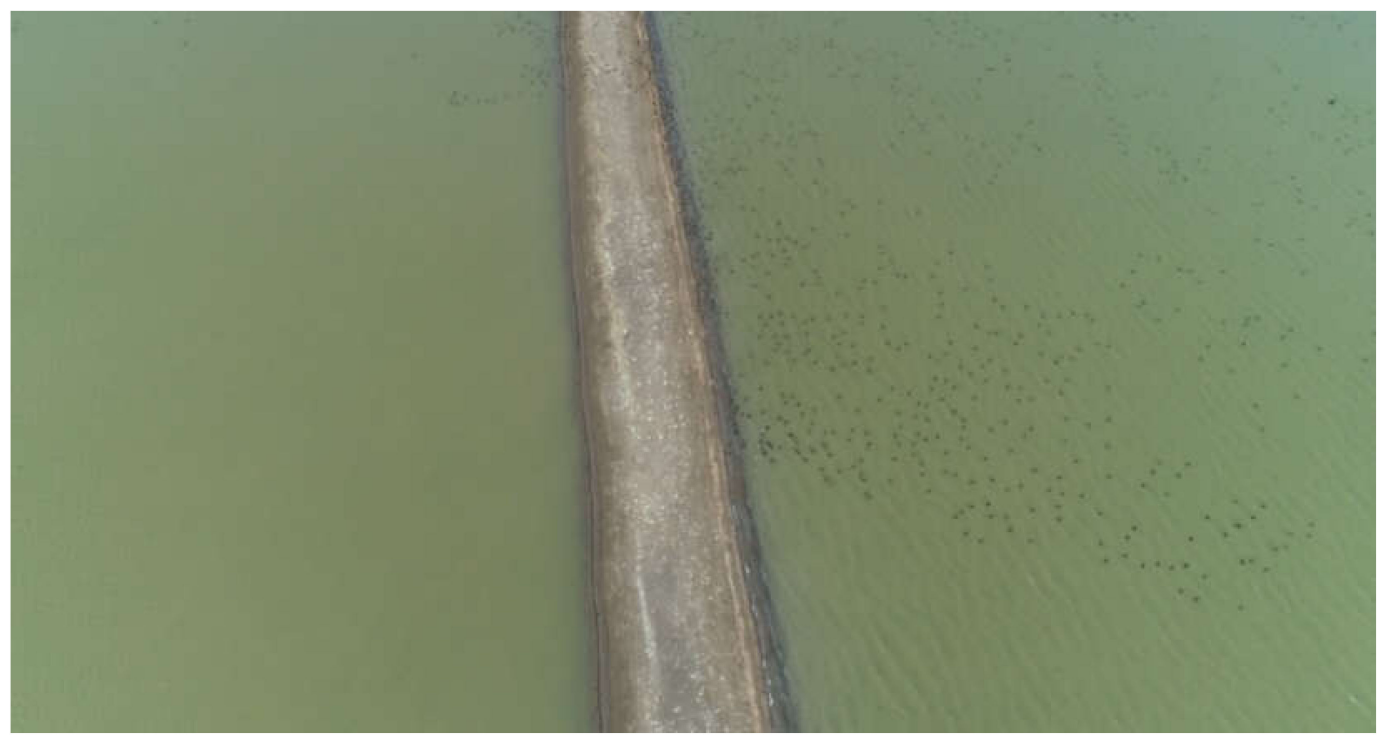

Figure 1. Example of perspective of waterfowl on water as seen from the drone. These waterfowl are primarily Grey Teal (Anas gracilis).

We analyzed the video using a custom program written in MATLAB (Mathworks, Natick, MA, USA) with the additional automated driving, computer vision, and image processing toolboxes. For each video, an observer went through all videos manually and identified and tagged all waterfowl seen. The total area of the dam covered by the field-of-view of the camera was calculated and the counts were adjusted for sampling effort.

\subsection{Statistical Analysis}

Differences between counts of waterfowl based on videos from the drone or from an observer on the ground (Table 1) were analyzed using Bayesian multilevel models (BMLM). 
Since each waterbody was surveyed by both the drone and a ground observer, individual waterbodies were included as a grouping-level effect. Population-level effects included survey method (ground or drone), waterfowl group size, and waterbody type (small dams $<5$ ha, medium dams $<10$ ha, large dams $<30$ ha, extra-large dams $30+$ ha, wastewater treatment ponds). In addition, differences in the total number of species detected were also examined. For the comparison of group size, the data were transformed to a standardized difference (S) between the drone count and the ground count using the equation:

$$
S=\frac{y_{-}(i, \text { drone })-y_{-}(i, \text { ground })}{y_{-}(i, \text { drone })}
$$

where $y_{-}(i, d r o n e)$ was the estimated group size for dam $i$ using the drone, and $y_{-}(i, g r o u n d)$ the estimate based on the ground count for dam $i$. To aid comparison, group sizes were categorized as either "small" (<100), "medium" (100-500), "medium-high" (501-1000), or "high" (1000+). The standardized difference data were assumed to follow a Gaussian distribution.

Models were fitted using four chains, each with 10,000 iterations and the first 1000 iterations used for warm-up. Convergence was assessed using Gelman and Rubin's convergence diagnostic $(\hat{R}<1.1 ;[14])$. The goodness-of-fit of individual models was assessed using conditional $R^{2}$, which can be interpreted as the variance explained by the entire model [15]. Model comparisons were done using Watanabe-Akaike information criterion (WAIC [16]).

The fitted models took the following form:

$$
\begin{gathered}
y_{i j} \sim \operatorname{Poisson}\left(\lambda_{i j}\right) \\
\lambda_{i j}=\alpha+\alpha_{\text {waterbody }[i]}+\beta_{i j} x_{i j} \\
\alpha \sim \operatorname{Student\_ t}(3,0,1) \\
\alpha_{\text {waterbody }} \sim \operatorname{Student\_ t}(3,0,1) \\
\beta \sim \text { Half Cauchy }(0,10)
\end{gathered}
$$

where the total count of waterfowl on waterbody $i$ using survey method $j$ is modeled as coming from a Poisson distribution with an expected count $\lambda_{i j}$. Following the recommendations of Gelman et al. [17], we used weakly informative priors for the intercepts and the slopes of the regressions. On average, the Cauchy distribution outperforms the normal because it allows for occasional large coefficients while still performing a reasonable amount of shrinkage for coefficients near zero, while the Student $t$-distribution can provide minimal prior information but still allows for robust inference because of its flat-tailed distribution. Except for the analysis of the number of species that were detected, all models assumed that the count data $y_{i j}$ followed a Poisson distribution. For the analysis of species identification, the data were assumed to follow a log-normal distribution.

All statistical analyses were carried out in R [18]. Data were prepared for analysis using the package tidyverse [19]. Multilevel models were fitted to the data using the package brms [20]. Plots were created using ggplot2 [21] and tables were formatted using sjPlot [22]. 
Table 1. Total counts of species by waterbody type and survey method (2018-2020).

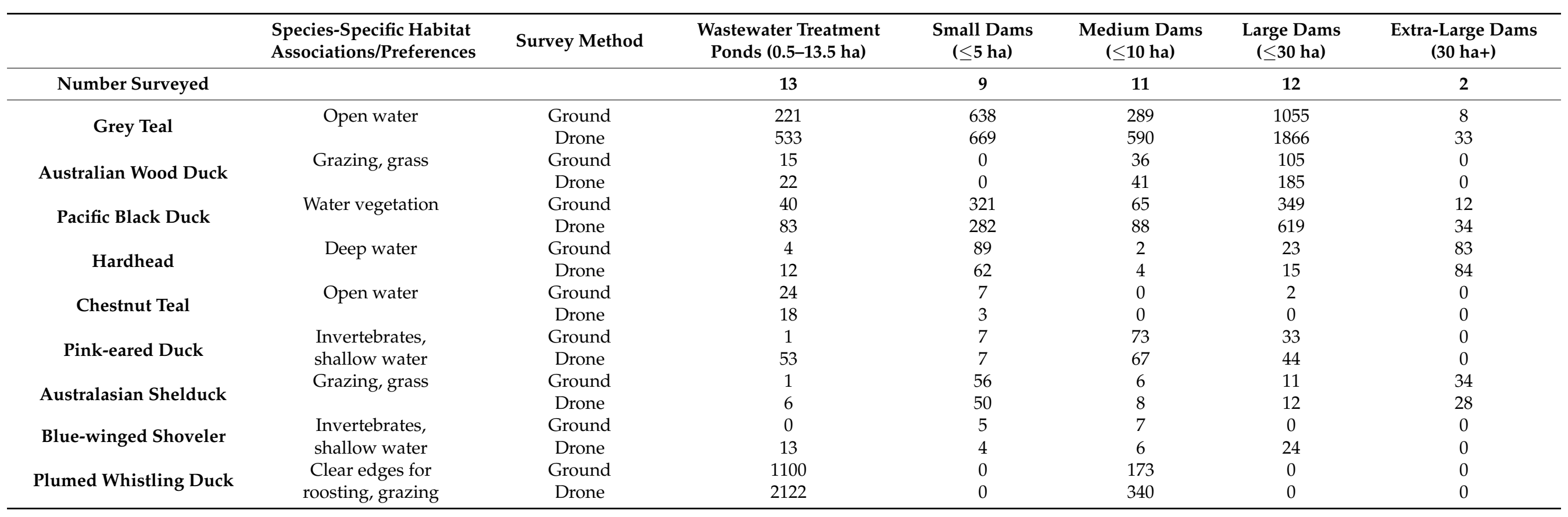




\section{Results}

\subsection{Comparison of Drone and Ground Counts of Waterfowl}

In total, 15,171 ducks were counted (Table 1). Comparisons of total counts-and not considering other covariates that might affect the counts-indicated that on any waterbody that was surveyed, there was a high likelihood that the drone count was greater than the ground count (approximately 36\% greater, $\beta$-ground $=0.64$ [0.62-0.66]) (Figure 2). The conditional $\mathrm{R}^{2}$ indicated that the model provided a good fit to the data $\left(\mathrm{R}^{2}=0.973\right)$.

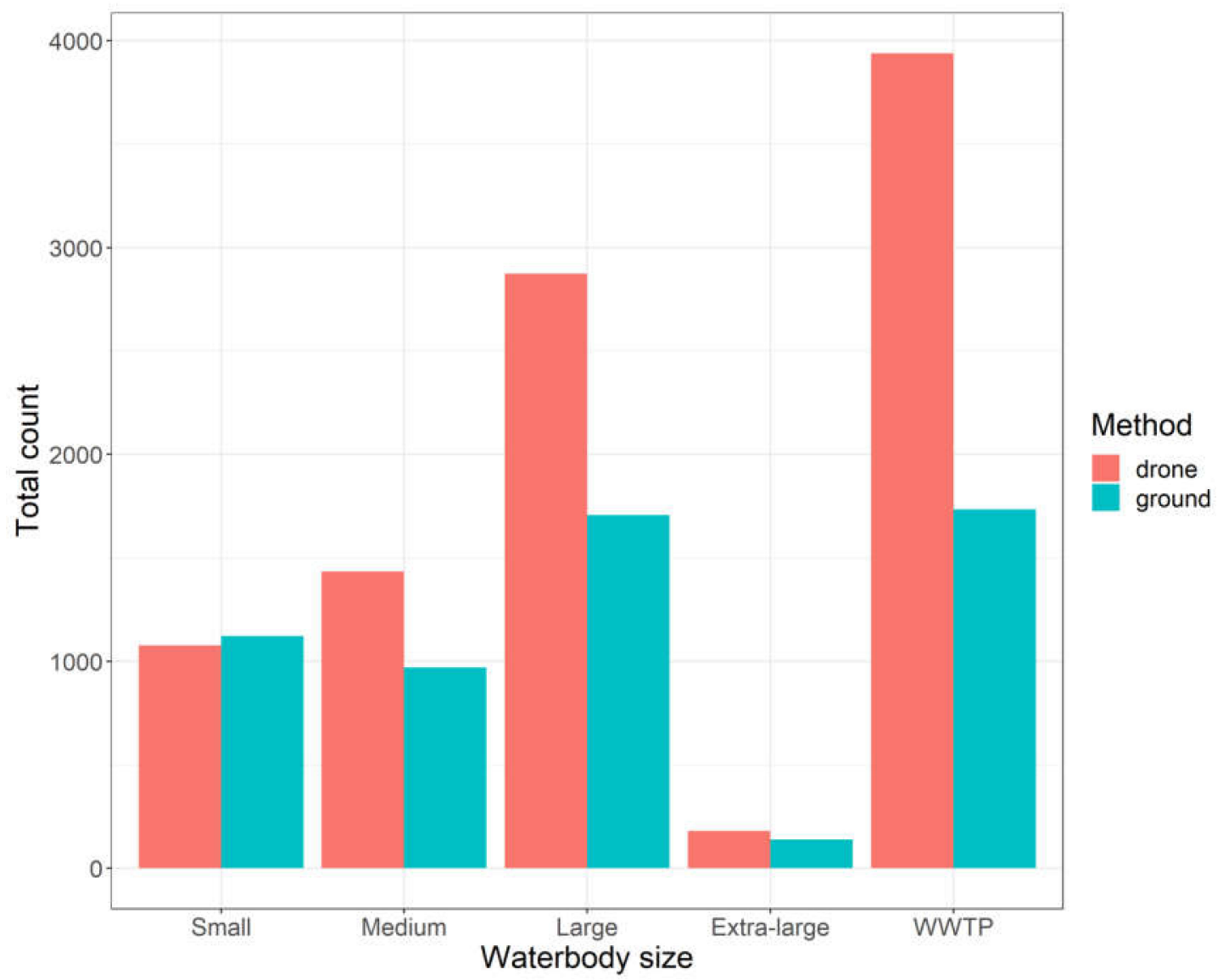

Figure 2. Comparison of total counts of waterfowl using two survey methods (drone and ground) across five different waterbody types.

\subsection{Comparison of Drone and Ground Counts Relative to Group Size}

Relative to counts using the drone, on average the ground counts tended to underestimate the number of waterfowl in a group (Table 2 and Figure 3). However, there was large variation in the data with some comparisons very close, while for others the ground count grossly underestimated the drone count (range: Low, -0.652-0.348; medium, -5.698-0.333; medium-high, -1.090--0.931; and high, -1.021--0.475). 
Table 2. The mean (estimate) and standard deviation (SD) of the posterior distribution of a model examining the standardized difference in group size estimated using the drone or ground count. Lower 95\% credible interval (CI) and upper 95\% CI are the two-sided credible intervals of the estimates based on quantiles. The large error estimates and wide credible intervals are a consequence of the large variability in group size estimated using the different survey methods. The number counted using the drone acted as the reference category.

\begin{tabular}{ccccc}
\hline Group Size & Estimate & SD & Lower 95\% CI & Upper 95\% CI \\
\hline Low & 0.862 & 1.329 & 0.492 & 1.506 \\
Medium & 0.255 & 1.445 & 0.125 & 0.529 \\
Medium-high & 0.385 & 2.801 & 0.051 & 2.958 \\
High & 0.489 & 2.767 & 0.068 & 3.589 \\
\hline
\end{tabular}

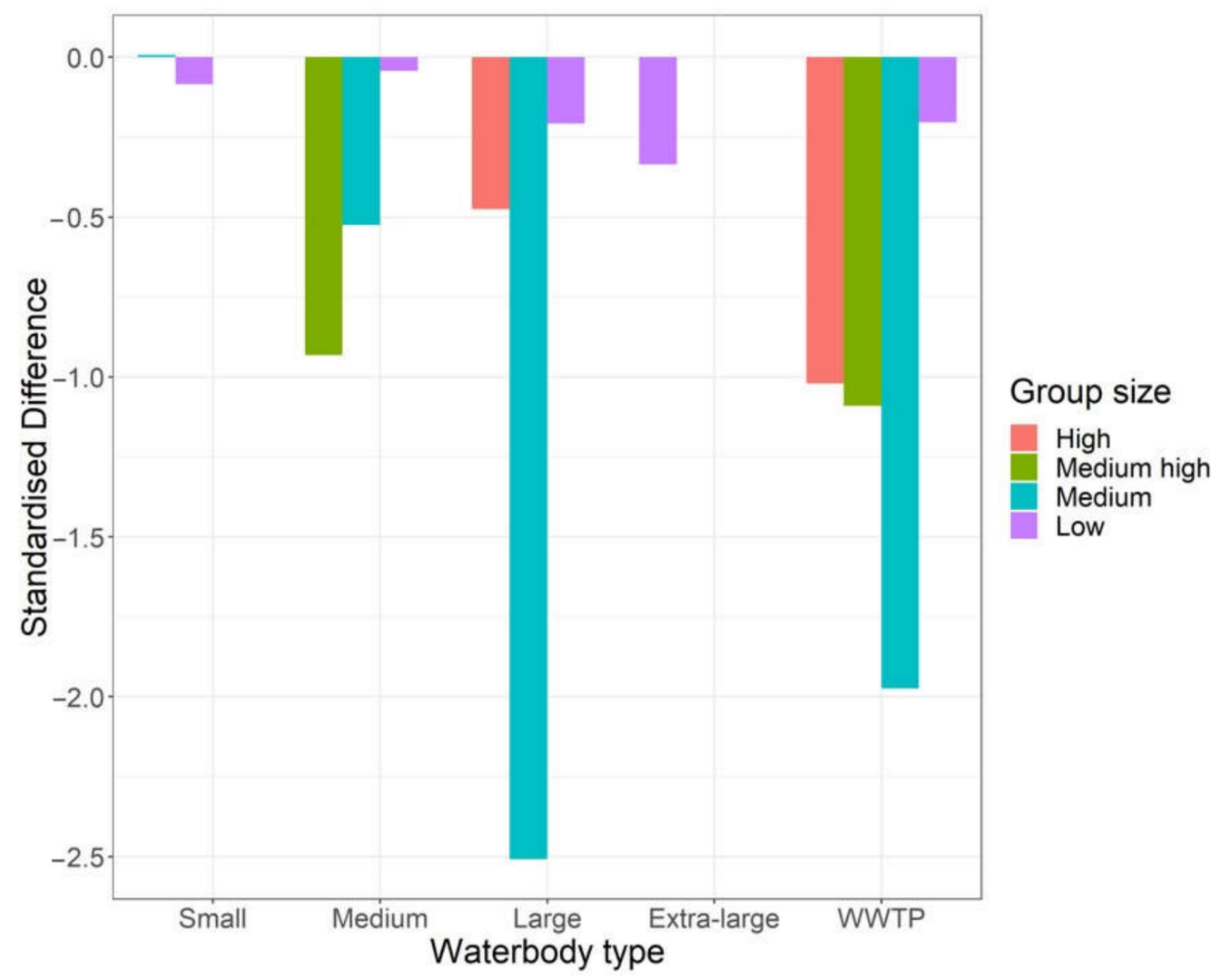

Figure 3. The standardized difference between group sizes estimated using drone- and ground-based survey methods.

\subsection{Comparison of Drone and Ground Counts Across Different Waterbody Types}

Waterbody type had an influence on the total counts of waterfowl (Table 3). A comparison of models that included the population-level effects waterbody type and survey method without an interaction term, compared with the same model that included an interaction between the variables, indicated that the model with the interaction had greater support from the data (WAIC with interaction $=1741.8$ vs. WAIC without interaction $=2015.5$ ). The ground counts at extra-large, medium, and large dams and wastewater treatment ponds were proportionately less than counts using a drone, with mean $=0.74$, $0.65,0.57$, and 0.51 [credible interval (CI): 0.59-0.94, 0.58-0.73, 0.52-0.63, 0.46-0.56], respectively. The exceptions were counts of waterfowl on small dams, which did not depend on the survey method. The model that included the interaction fitted the data well with a conditional $\mathrm{R}^{2}=0.98$. 
Table 3. The mean (estimate) and standard deviation (SD) of the posterior distribution of a model examining the interaction between the counts of waterfowl observed on each waterbody type and the survey method. Lower 95\% CI and upper 95\% CI are the two-sided credible intervals of the estimates based on quantiles. The large error estimates and wide credible intervals are a consequence of the large variability in the number of waterfowl counted on each waterbody type.

\begin{tabular}{ccccc}
\hline $\begin{array}{c}\text { Survey Method: } \\
\text { Waterbody Type }\end{array}$ & Estimate & SD & Lower 95\% CI & Upper 95\% CI \\
\hline Drone: Small & 0.995 & 1.542 & 0.426 & 2.339 \\
Ground: Small & 1.038 & 1.542 & 0.442 & 2.446 \\
Drone: Medium & 1.013 & 1.534 & 0.430 & 2.354 \\
Ground: Medium & 0.686 & 1.534 & 0.291 & 1.596 \\
Drone: Large & 1.401 & 1.516 & 0.614 & 3.182 \\
Ground: Large & 0.833 & 1.516 & 0.364 & 1.892 \\
Drone: Extra-large & 1.145 & 1.757 & 0.369 & 3.514 \\
Ground: Extra-large & 0.876 & 1.759 & 0.281 & 2.690 \\
Drone: WWTP & 1.509 & 1.519 & 0.664 & 3.425 \\
Ground: WWTP & 0.796 & 1.520 & 0.350 & 1.809 \\
\hline
\end{tabular}

\subsection{Comparison of Species Detected During Drone and Ground Counts}

The number of species that could be identified on each waterbody type was similar regardless of survey method (Figure 4). The difference between species identifications made by drone (the reference category) and ground observer were small and the $95 \%$ credible interval $(95 \% \mathrm{CI})$ included zero $\left(\beta \_\right.$ground $\left.=0.01[-0.07,0.09]\right)$. The overall model that only included the survey method as the explanatory variable provided a reasonable fit to the data $\left(R^{2}=0.728\right)$.

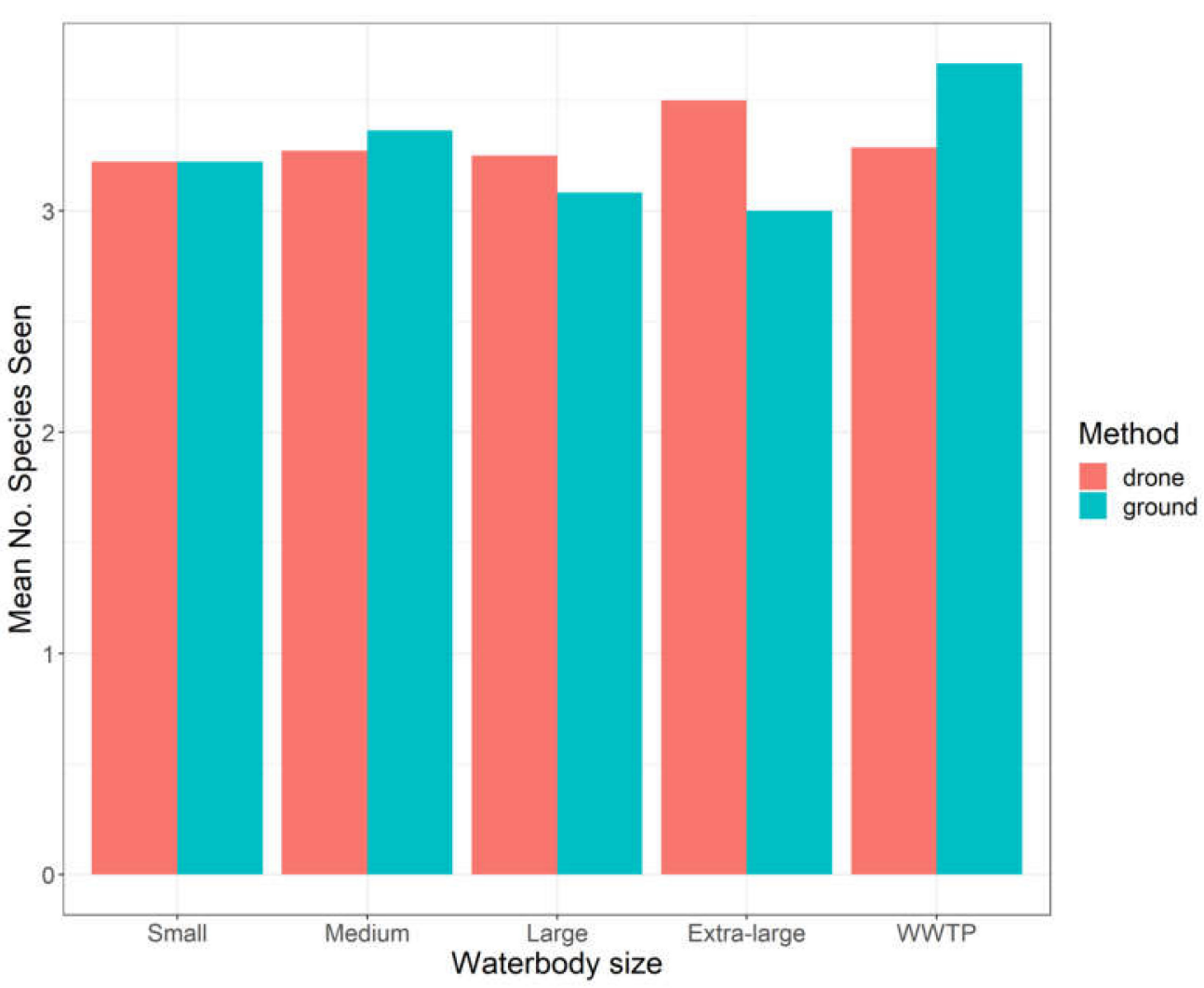

Figure 4. The mean number of species of waterfowl that were identified on each waterbody type using the two survey methods. 
There was strong evidence that survey method influences the count of most species of waterfowl but there are some exceptions (Table 4). The comparison of survey methods indicated that ground counts underestimated the abundance of Grey Teal, Australian Wood Duck, Pacific Black Duck, Pink-eared Duck, Blue-winged Shoveler, and Plumed Whistling Duck, by an average 38\%. In contrast, ground counts of Hardhead, Chestnut Teal, and Australasian Shelduck tended to be higher than the drone counts but the differences were not large and the credible intervals for each species included 1 , indicating that there was not strong evidence that counts were significantly different.

Table 4. Results of a multivariate analysis examining the influence of survey method (drones vs. ground count) on counts of nine species of waterfowl. The mean (estimate) and standard deviation (SD) of the posterior distribution of a model of the count for each species, and the lower $95 \% \mathrm{CI}$ and upper $95 \% \mathrm{CI}$ are the two-sided credible intervals of the estimates based on quantiles. The number counted using the drone acted as the reference category.

\begin{tabular}{ccccc}
\hline Species & Estimate & SD & Lower 95\% CI & Upper 95\% CI \\
\hline Grey Teal & 0.612 & 1.025 & 0.582 & 0.643 \\
Australian Wood Duck & 0.687 & 1.094 & 0.575 & 0.821 \\
Pacific Black Duck & 0.809 & 1.038 & 0.751 & 0.871 \\
Hardhead & 1.153 & 1.105 & 0.947 & 1.405 \\
Pink-eared Duck & 0.664 & 1.129 & 0.522 & 0.843 \\
Chestnut Teal & 1.536 & 1.250 & 0.994 & 2.391 \\
Australasian Shelduck & 1.037 & 1.143 & 0.795 & 1.350 \\
Blue-winged Shoveler & 0.379 & 1.277 & 0.232 & 0.605 \\
Plumed Whistling Duck & 0.567 & 1.031 & 0.533 & 0.603 \\
\hline
\end{tabular}

Analysis of drone vs. ground count data are shown in Supplementary Materials.

\section{Discussion}

\subsection{Comparison of Drone and Ground Counts of Waterfowl}

Drone surveys more often resulted in larger counts of ducks on waterbodies compared to traditional ground surveys. Our results align with other comparisons between survey methods including counts of colonial nesting seabird colonies [23] and nesting shorebirds [9] where drone surveys resulted in more accurate counts compared to ground counts. The main advantage we observed is that drone surveys allowed for a better perspective from a higher vantage point, especially when some birds were hiding within vegetation or sitting on banks, which is likely to have contributed to the higher drone counts.

\subsection{Comparison of Drone and Ground Counts Relative to Group Size}

Large flocks of non-nesting birds can be difficult to count from the ground as they tend to fly around and are easily disturbed. We found the difference in drone and ground counts was more pronounced when flock sizes were greater than 100. Analysis of video footage from the drone allowed us to rewatch and pause on larger flocks which made them easier to count and identify and resulted in more accurate counts. Similarly, counts of replica seabirds from drone images were more accurate than counts performed out in the field [15]. An additional benefit of capturing videos and images is that they can be archived allowing for repurposing data for additional analyses in future.

\subsection{Comparison of Drone and Ground Counts across Different Waterbody Types}

We found drone counts were more accurate when the size of the waterbody was greater than 5 ha in size. Ground counts were carried out at a single point with a good vantage point across the waterbody, but for larger waterbodies, there are more likely to be areas that will not be visible to a ground observer. The presence of vegetation in particular reduces the visibility of ducks when carrying out ground-based surveys [24]. Wastewater treatment ponds are known to be readily occupied by ducks, with the settling 
ponds providing a rich source of macroinvertebrates [25-27], but often the layout of the ponds means ducks can be easily missed when surveying on ground. For example, Plumed Whistling Ducks are commonly seen at WWTPs but are usually congregated on the banks in large numbers, which can make them difficult to count on ground.

\subsection{Comparison of Species Detected During Drone and Ground Counts}

The number of species that could be identified on each waterbody type was similar regardless of survey method, but drone counts of most species (Grey Teal, Australian Wood Duck, Pacific Black Duck, Pink-eared Duck, Blue-winged Shoveler, and Plumed Whistling Duck) were on average 38\% higher than the ground counts. Ground counts were higher than drone surveys for three species of waterfowl: Hardhead, Chestnut Teal, and Australasian Shelduck. Australasian Shelduck were observed to be particularly sensitive to the presence of the drone and would often fly off the waterbody as the drone was launched. Non-nesting Ibis species (Straw-necked Ibis, Threskiornis spinicollis and Australian White Ibis, Threskiornis moluccus) were also noted as being especially sensitive to the presence of a drone in the air. Hardhead are a diving duck species and can sometimes be difficult to count when they are actively foraging. If they are not on the surface of the water when the drone flies over, it is likely they will be missed on the drone video. There is a similar issue for low-level fixed-wing aerial surveys of waterbirds where diving species such as grebes dive under the water when the plane flies overhead [28]. Female Chestnut Teal can be difficult to distinguish from Grey Teal on the ground unless the observer can clearly see the darker grey throat, or she is near a male mate. This is likely to account for underestimates of this species.

\subsection{Reactions of Birds to Drones}

In some cases, we found ducks flew off when the drone was airborne and could only be surveyed on ground. In other cases, ducks stayed on the water and showed little reaction to the drone flying overhead (Figure 1), although Australia shelduck were the exception. We found ducks tended to react together as a flock. If a few were flighty, then the rest of the flock was more likely to take off. Some ducks would also have been exposed to shooting for pest mitigation, so the presence of a human made them more wary. Previous surveys of non-breeding ducks also observed a flush response in the presence of a drone with larger flocks more likely to react compared to smaller flocks [29]. Drone flight speed, color of the drone, and repeated flyovers did not appear to affect Mallards (Anas platyrhynchos), Greater Flamingos (Phoenicopterus roseus), or Common Greenshanks (Tringa nebularia) [17]. However, the angle of the approach was identified as the factor most likely to influence behavioral responses to the drone, and at an optimal angle, the drone could be flown as close as $4 \mathrm{~m}$ without disturbing the bird [30]. The use of drones will inevitably cause some short-term disturbance to waterbirds, but drone flights of short duration involving quick flyovers do not appear to cause any more disturbance than would be caused by the presence of a ground observer. However, as the use of drones for bird surveys increases, the development of ethical standards for drone surveys is paramount [30]. This will be especially important for surveys of breeding birds where disturbance has the potential to cause birds to abandon nests or chicks.

\subsection{Drawbacks Related to the Use of Drones}

The use of drones for wildlife surveys does has some drawbacks including initial cost of equipment and increased labor requirements for pre-survey preparation and postsurvey image processing. Drone surveys require lodgment of flight plans prior to the flight, charging batteries, setting up and packing up the drone, flying the grid pattern, and processing the resulting videos. Processing of imagery following surveys can be time consuming and laborious [24]. There have been recent advancements related to deep machine learning for object detection which have shown promise for identifying waterbirds in different habitats $[10,11]$. The reliability of these systems will be reliant on 
a large number of manually identified images of waterbirds in a range of stances (flying, swimming, nesting, resting on bank, within vegetation) in a range of different habitats.

Drones are becoming increasingly more cost effective with a range of drones available to suit all budgets [31]; however, there is an initial outlay for equipment in addition to the added costs of training. As a general rule, more expensive drones and cameras will provide better image quality, as is usually required for accurate species identification. We found video was better for identification of waterfowl, but the frames per second (fps) rate of the camera needed to be at least 60 . Previous surveys have found ground resolution needs to be at least $1 \mathrm{~cm} /$ pixel for accurate identification of waterbird species [32].

As with any technology, technical glitches happen and these issues take extra time to sort out, and in remote areas, troubleshooting can be challenging. In areas with limited phone and internet reception, there can be difficulty obtaining satellite images required to set up the automatic flight paths. The weather can have a major influence on the quality of the video and the performance of the drone and although the Matrice 210 can be flown in light showers, we found the water droplets on the camera lens greatly reduced video quality. Similarly, windy conditions caused some shaking of the drone, resulting in less suitable video footage (this was more of an issue for the $1.4 \mathrm{~kg}$ Phantom 4 Pro drone compared to the $4.9 \mathrm{~kg}$ Matrice 210). Overcast conditions can make the ducks more difficult to identify, especially if they do not fly off the water (allowing for identification from distinctive wing patterns).

\section{Conclusions}

Drones proved to be an effective tool for surveying non-breeding waterfowl at our sites with drone-derived counts being more accurate than ground counts. In particular, we obtained better counts of waterfowl for larger groups of birds on larger waterbodies from the drone videos. Ideally, multiple survey methods should be used where possible to yield more accurate counts by accounting for missed animals and harder-to-detect species. It is unlikely drones will be a suitable replacement to ground surveys for all bird species in all habitat types, but for waterfowl using artificial waterbodies, drone surveys are a better option.

Supplementary Materials: Datasets and scripts are available online https:/ / www.mdpi.com/2504 $-446 \mathrm{X} / 5 / 1 / 5 / \mathrm{s} 1$.

Author Contributions: Conceptualization, S.J.D., M.V., P.O., and S.R.M.; methodology, S.J.D., M.V., and S.R.M.; fieldwork, S.J.D., M.V., P.O., and S.R.M.; formal analysis, S.R.M. and S.J.D.; data curation, S.J.D.; writing—original draft preparation, S.J.D., M.V., and S.R.M.; writing-review and editing, S.J.D., M.V., P.O., and S.R.M. All authors have read and agreed to the published version of the manuscript.

Funding: This research was funded by the NSW State Government Department of Primary Industries Game Licensing Unit.

Institutional Review Board Statement: Not applicable.

Informed Consent Statement: Not applicable.

Data Availability Statement: The data presented in this study are openly available in [Shannon J. Dundas].

Acknowledgments: The authors would like to thank landowners and councils for proving access to waterbodies on private property. Approvals for all work with animals (drone and ground surveys) were approved by the Orange Animal Ethics Committee at the Department of Primary Industries-Animal Research Authority ORA 18/21/008 and NSW Parks and Wildlife Service scientific license SL102055. All authors are licensed drone pilots with a current Remote Pilot License (RePL) and Aircraft Radio Operator Certificate (AROC) as required by the Australian Civil Aviation Safety Authority (CASA).

Conflicts of Interest: The authors declare no conflict of interest. 


\section{References}

1. Linchant, J.; Lisein, J.; Semeki, J.; Lejeune, P.; Vermeulen, C. Are unmanned aircraft systems (UASs) the future of wildlife monitoring? A review of accomplishments and challenges. Mammal Rev. 2015, 45, 239-252. [CrossRef]

2. Van Gemert, J.C.; Verschoor, C.R.; Mettes, P.; Epema, K.; Koh, L.P.; Wich, S. Nature Conservation Drones for Automatic Localization and Counting of Animals. In Proceedings of the Computer Vision-ECCV 2014 Workshops, Zurich, Switzerland, 6-7 September 2014; pp. 255-270.

3. Afán, I.; Máñez, M.; Díaz-Delgado, R. Drone Monitoring of Breeding Waterbird Populations: The Case of the Glossy Ibis. Drones 2018, 2, 42. [CrossRef]

4. $\quad$ Rodgers, J.A.; Linda, S.B.; Nesbitt, S.A. Comparing Aerial Estimates with Ground Counts of Nests in Wood Stork Colonies. J. Wildl. Manag. 1995, 59, 656-666. [CrossRef]

5. Chabot, D.; Craik, S.R.; Bird, D.M. Population census of a large Common Tern colony with a small unmanned aircraft. PLoS ONE 2015, 10, e0122588. [CrossRef]

6. Rush, G.P.; Clarke, L.E.; Stone, M.; Wood, M.J. Can drones count gulls? Minimal disturbance and semiautomated image processing with an unmanned aerial vehicle for colony-nesting seabirds. Ecol. Evol. 2018, 8, 12322-12334. [CrossRef]

7. Jachmann, H. Estimating Abundance of African Wildlife: An Aid to Adaptive Management; Springer Science \& Business Media: New York, NY, USA, 2012.

8. Ivošević, B.; Han, Y.-G.; Cho, Y.; Kwon, O. The use of conservation drones in ecology and wildlife research. J. Ecol. Environ. 2015, 38, 113-118. [CrossRef]

9. Valle, R.G.; Scarton, F. Drones improve effectiveness and reduce disturbance of censusing common redshanks Tringa totanus breeding on salt marshes. Ardea 2020, 107, 275-282. [CrossRef]

10. Hong, S.-J.; Han, Y.; Kim, S.-Y.; Lee, A.-Y.; Kim, G. Application of deep-learning methods to bird detection using unmanned aerial vehicle imagery. Sensors 2019, 19, 1651. [CrossRef]

11. Francis, R.J.; Lyons, M.B.; Kingsford, R.T.; Brandis, K.J. Counting mixed breeding aggregations of animal species using drones: Lessons from waterbirds on semi-automation. Remote Sens. Ecol. Conserv. 2020, 12, 1185. [CrossRef]

12. Scally, J.; Schwarzman, R.; Stirling, K.; McGuinness, S. Riverina Murray Agricultural Industries Final Report for Department of Planning and Environment; RMCG: Bendigo, Australia, 2016.

13. Dundas, S.J.; Vardanega, M.; McLeod, S. 2019-2020 Annual Waterfowl Quota Revised report to the Game Licensing Unit, NSW Department Primary Industries-August 2019; Vertebrate Pest Research Unit NSW Department of Primary Industries: Orange, Australia, 2019.

14. Gelman, A.; Carlin, J.B.; Stern, H.S.; Dunson, D.B.; Vehtari, A.; Rubin, D.B. Bayesian Data Analysis, 3rd ed.; CRC Press: Boca Raton, FL, USA, 2013.

15. Nakagawa, S.; Schielzeth, H. A general and simple method for obtaining R2 from generalized linear mixed-effects models. Methods Ecol. Evol. 2013, 4, 133-142. [CrossRef]

16. Watanabe, S. Asymptotic equivalence of Bayes cross validation and widely applicable information criterion in singular learning theory. J. Mach. Learn. Res. 2010, 11, 3571-3594.

17. Gelman, A.; Jakulin, A.; Pittau, M.G.; Su, Y.-S. A weakly informative default prior distribution for logistic and other regression models. Ann. Appl. Stat. 2008, 2, 1360-1383. [CrossRef]

18. R Core Team. R: A Language and Environment for Statistical Computing; R Foundation for Statistical Computing: Vienna, Austria, 2020; Available online: https:/ / www.R-project.org/ (accessed on 16 October 2020).

19. Wickham, H.; Averick, M.; Bryan, J.; Chang, W.; McGowan, L.D.; François, R.; Grolemund, G.; Hayes, A.; Henry, L.; Hester, J.; et al. Welcome to the tidyverse. J. Open Source Softw. 2019, 4, 1686. [CrossRef]

20. Bürkner, P.-C. brms: An R package for bayesian generalized linear mixed models using Stan. J. Stat Softw. 2017, 80, 1-28. [CrossRef]

21. Wickham, H. ggplot2: Elegant Graphics for Data Analysis; Springer: New York, NY, USA, 2016.

22. Lüdecke, D. sjPlot: Data Visualization for Statistics in Social Science; R package Version 2.8.5; 2020. Available online: https: / /CRAN.R-project.org / package=sjPlot \T1 \textgreater \{\} (accessed on 16 October 2020).

23. Hodgson, J.C.; Baylis, S.M.; Mott, R.; Herrod, A.; Clarke, R.H. Precision wildlife monitoring using unmanned aerial vehicles. Sci. Rep. 2016, 6, 22574. [CrossRef]

24. Pöysä, H.; Kotilainen, J.; Väänänen, V.-M.; Kunnasranta, M. Estimating production in ducks: A comparison between ground surveys and unmanned aircraft surveys. Eur. J. Wildl. Res. 2018, 64, 74. [CrossRef]

25. Loyn, R.H.; Swindley, R.J.; Stamation, K. Waste water not wasted: The Western treatment plant as a habitat for waterfowl. Vic. Nat. 2014, 131, 147-149.

26. Murray, C.G.; Kasel, S.; Szantyr, E.; Barratt, R.; Hamilton, A.J. Waterbird use of different treatment stages in waste-stabilisation pond systems. Еmu 2014, 114, 30-40. [CrossRef]

27. Bélanger, L.; Couture, R. Use of man-made ponds by dabbling duck broods. J. Wildl. Manag. 1988, 52, 718-723. [CrossRef]

28. Gilbert, A.D.; Jacques, C.N.; Lancaster, J.D.; Yetter, A.P.; Hagy, H.M. Disturbance caused by aerial waterfowl surveys during the nonbreeding season. J. Wildl. Manag. 2020, 84, 1063-1071. [CrossRef]

29. Jarrett, D.; Calladine, J.; Cotton, A.; Wilson, M.W.; Humphreys, E. Behavioural responses of non-breeding waterbirds to drone approach are associated with flock size and habitat. Bird Study 2020, 1-7. [CrossRef] 
30. Vas, E.; Lescroël, A.; Duriez, O.; Boguszewski, G.; Grémillet, D. Approaching birds with drones: First experiments and ethical guidelines. Biol. Lett. 2015, 11, 20140754. [CrossRef]

31. Iv, G.P.J.; Pearlstine, L.G.; Percival, H.F. An Assessment of Small Unmanned Aerial Vehicles for Wildlife Research. Wildl. Soc. Bull. 2006, 34, 750-758. [CrossRef]

32. Drever, M.C.; Chabot, D.; O'Hara, P.D.; Thomas, J.D.; Breault, A.; Millikin, R.L. Evaluation of an unmanned rotorcraft to monitor wintering waterbirds and coastal habitats in British Columbia, Canada. J. Unmanned Veh. Syst. 2015, 3, 256-267. [CrossRef] 